\section{Evidence-Based Health Care. A CASP CD-ROM and Workbook}

Update Software Ltd. Price £79.99. ISBN 1-901868-41-9

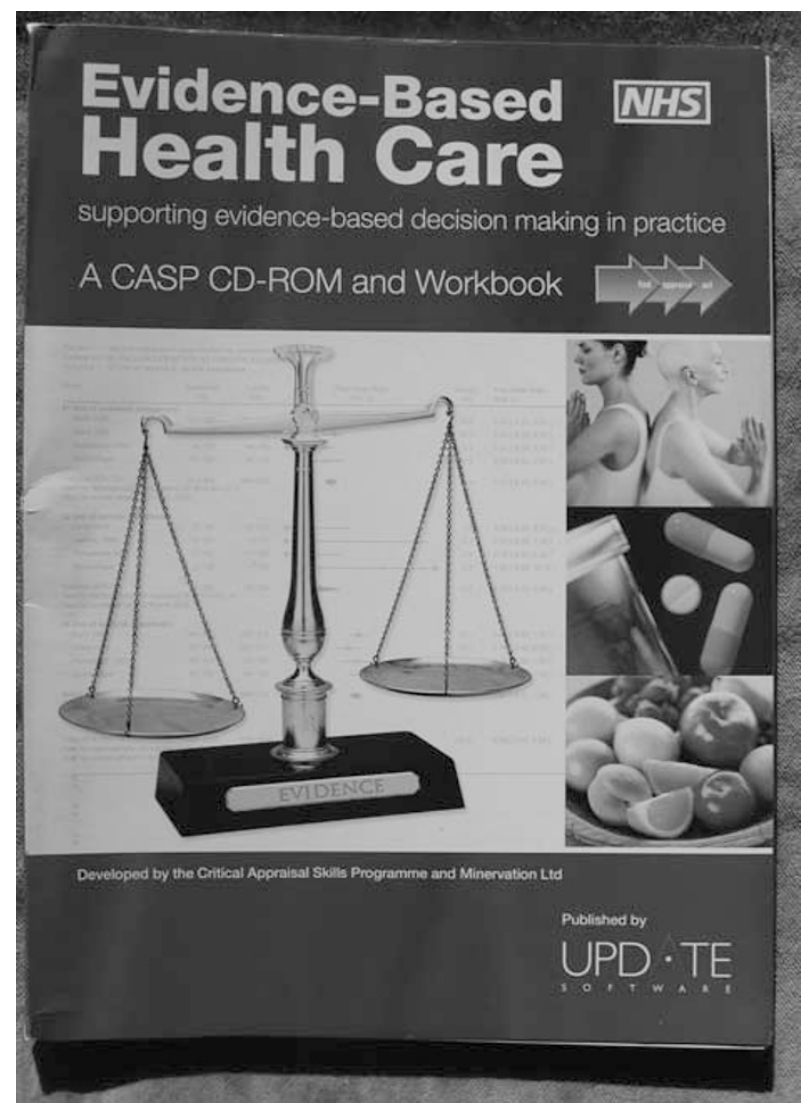

I reviewed the first edition of this CD-ROM and workbook in the second issue of Evidence-based Dentistry, back in 1999. ${ }^{1}$ The original version of which consisted of five main areas:

- An introduction to evidence-based healthcare

- Asking clinical questions

- Finding evidence

- Randomised controlled trials

- Systematic reviews

The latest edition has additional sections about qualitative research, economic evaluation and acting on evidence. The section on asking clinical questions has understandably moved into "Finding evidence" - it being much easier to find something when you know exactly what you are looking for. This results in a CD-ROM that now has seven main parts, containing 30 structured tutorials. Each of the latter takes between 20 and 30 minutes to work through.

The workbook contains some background information, copies of the original article for critical appraisal, and appraisal worksheets developed by the Critical Appraisal Skills Programme (see www.phru.nhs.uk/casp/casp.htm), based within Learning and Development at the Public Health Resource Unit in Oxford, UK.

The CD-ROM has retained its easy-to-navigate format, and the instructions for use for people unfamiliar with this type of product are clear (Figure 1). The Tools section of the menu allows the reader to create a record of continuing professional development (CPD): this indicates which modules and what proportion of the overall package you have completed. As the sections have clear aims, I believe that the package would fulfill the General Dental Council's requirements for verifiable $\mathrm{CPD}$.

On opening the program, the first display appears only in the centre of the screen. I would like the facility to maximise this to full-screen, but this is a relatively small gripe about what is a very useful package. This version is much improved on the earlier ones. The "Finding evidence" section has undergone major changes and now includes information on a wide range of databases (Clinical Evidence, Cochrane Library, Prodigy, Evidence-based Medicine Reviews, British Nursing Index, Cinhal (Cumulative Index to nursing and Allied Health Literature), Embase, Medline and PsychInfo) and search engines. Other useful additions are the section page-maps (Figure 2) which allow the user to jump to specific areas of the course, while also illustrating the content you have already worked through and what is still to cover.

Having delivered critical appraisal workshops for a number of years, one of the common feedback points are problems related to understanding statistical terms. A strong element of this package is the simple way in which terms such as odds ratio, relative risk and

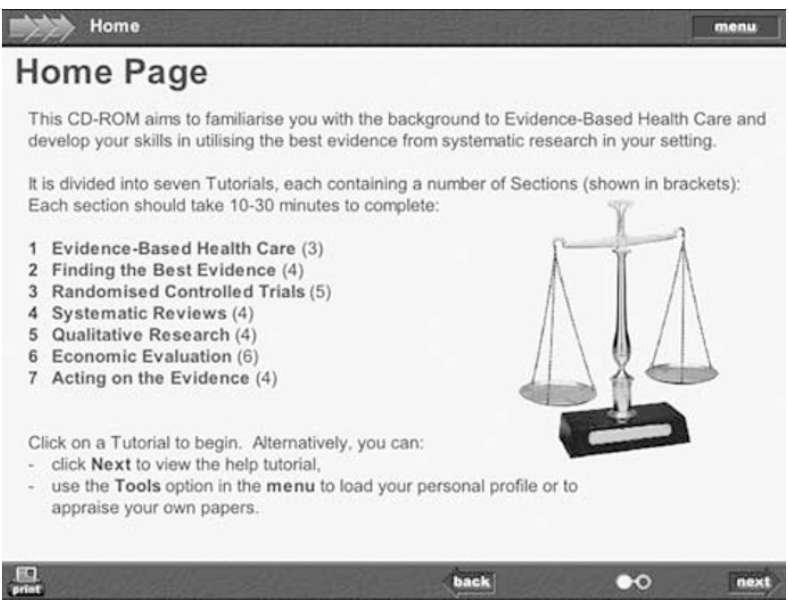

Figure 1. CD-ROM home page.

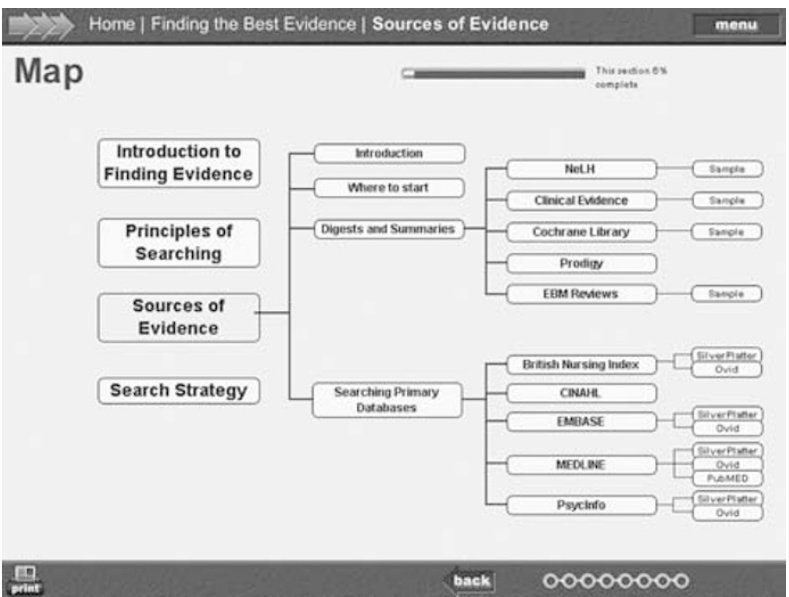

Figure 2. CD-ROM section maps. 
numbers needed-to-treat are explained and worked through using simple, easy-to-follow examples.

The package also contains a number of short video clips in which Dr Muir Gray (Director of the National Electronic Library), Professor John Geddes (Director of the Centre for Evidence-based Mental Health) and Professor Alistair Gray (Health Economics Research Centre of Oxford University) explain a number of the relevant concepts that are covered in text. These provide a break from the text-based structure of the overall package, although I did experience some minor problems while viewing some of them.

Even though the target audience for this package is general practitioners, nurses and allied health professionals, there is much of value for the dental team. This is because a large proportion of the core information is generic: question formulation, searching for evidence, and databases and search engines. Critical appraisal is also a generic skill, but the papers in the workbook for this subject, dealing with hypertension, pain relief, breast cancer care and economic analysis of hernia repair, although perhaps of interest to the dental team, lack direct relevance to everyday practice and would limit their use.

The final section of the workbook is on "Acting on evidence", the most difficult step in the evidence-based healthcare process, and includes an interesting and useful case study on implementing guidelines in primary care.

\section{Derek Richards}

Centre for Evidence-based Dentistry, Oxford, UK

1. Richards D. Interactive critical appraisal. Evid Based Dent 1999; 1:24.

Evidence-Based Dentistry (2005) 6, 108-109.

doi:10.1038/sj.ebd.6400368 